\title{
Effect of Ankle Joint Mobilization and FES on Change of Ankle Movement and the Quality of Gait in Patients with Hemiplegia
}

\begin{abstract}
This study was conducted to investigate the effect of FES and ankle mobilization on the ankle motion and the quality of gait of chronic hemiplegic patients with limited ankle joint motions. As research subjects, 24 chronic hemiplegic patients who could walk independently, regardless of assistive aids, were selected. Then, 8 subjects received mobilization randomly and 8 subjects received FES and 8 subjects received mobilization and FES, at the same time. The dorsiflexion PROM significantly increased in the group of mobilization therapy, mobilization and FES all together(p〈.01). There were statistically significant differences among the three groups $(p<.01)$. The $10 \mathrm{~m}$ walking test significantly decreased in the group of mobilization therapy, mobilization and FES all together(p〈.05). There were statistically significant differences among the three groups( $p\langle .01)$. The gait velocity significantly increased in the group of mobilization therapy, FES therapy, mobilization and FES all together(p<.05). There were statistically significant differences among the three groups(p<.01). The stride length significantly increased in the group of mobilization therapy, mobilization and FES all together(p〈.05). There were statistically significant differences among the three groups(p<.05). In conclusion, these findings demonstrate that rather than only using one treatment technique, applying mobilization and FES together brings a more satisfactory result to hemiplegic patients with limited ankle joint motions.
\end{abstract}

Key words: hemiplegic patients, ankle movement, mobilization, FES
Hyun Suk Lee ${ }^{a}$, Si Eun Park Sang Bin Lee ${ }^{c}$, Bo Kyoung Kim ${ }^{d}$, Hee Joon Shine, Hong Rae Kim Young Duk Choi', Kyung Ok Min ${ }^{\mathrm{b}}$

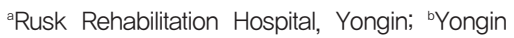
University, Yongin; 'Namseoul University, Cheonan; 'International University of Korea, Jinju; eKyungwoon University, Gumi; 'Kyungdong University, Goseong, Korea

Received : 29 May 2014

Revised : 19 June 2014

Accepted : 02 September 2014

Address for correspondence

Kyung Ok Min, PT, Ph.D

Department of Physical Therapy, Yongin University, 470 Samga-dong, Cheoingu, Yongin, Korea

Tel: 82-31-8020-2771

E-mail: komin@yongin.ac.kr

\section{INTRODUCTION}

Malignant neoplasm, cerebrovascular disease and heart disease are the three major causes of death and here, cerebrovascular disease, which is the second biggest cause of death, is found in 51.1 out of 100,000 people and has been reported to have the highest death rate as a single disease(1). Among various clinical features of stroke patients, ankle joint stiffness that causes motor disorders, stiffens the ankle joint due to the shortening of plantar flexors and imposes restrictions on the ankle joint motion by increasing the hypomobility of the joint and the resistance of the joint motion and decreasing the passive joint motion(2-4). Therefore, there is a need to develop a physical therapy intervention effective in improving the ankle joint motion to enhance gait abilities of chronic hemiplegic patients with stiffened and contractured ankle joints.

As studies on the ankle joint of chronic hemiplegic patients so far, there are 'The Effect of FES(Functional Electrical Stimulation) on the Gait of Stroke Patients(5)', 'The Effect of FES Applied to the Tibialis Anterior of Chronic Hemiplegic Patients on the Ankle Joint Motion(6)', 'The Influence of Mobilization with Active Movement Applied to the Ankle of Chronic Hemiplegic Patients on SpatioTemporal Variables of Lower Extremity and Gait(7)', 'The Influence of Ankle Strengthening Exercise and FES on Gait Activities and Balance Activities of Stroke Patients(8)', etc. But yet, there is no research to compare and analyze the effect of mobilization and 
FES, which are applied simultaneously.

Therefore, this study compared the application methods of mobilization and FES and analyzed the effect by applying them to chronic hemiplegic patients with limited ankle joint motions.

\section{METHODS}

\section{Subject}

This study targeted 24 stroke patients in $\mathrm{R}$ hospital located in S City, Gyeonggi-do, who suffered from gait disturbances caused by the foot drop and could independently walk over $20 \mathrm{~m}$, regardless of the application of walking assist devices. After dividing them into three groups of eight each, the experiments were conducted from November 2013 to May 2014.

The general characteristics of the study subjects are shown in 〈Table 1〉.

\section{Experiment protocols}

\section{Mobilization therapy}

Mobilization was applied by a therapist, who completed the Kaltenborn-Evjenth Orthopaedic Manipulation Upper and Lower Limb Therapy Course for 30 minutes a day and twice a week and for 4 weeks. The patients were asked to put the posterior side of the calf onto the treatment couch and the ankle joint in a resting position.

The calf was fixed on the treatment couch using a fixing belt. After wrapping the talus with a towing belt, a loop was made to be long enough to reach the floor. The therapist made the tiptoe touch the floor and moved the heel downward in a standing position after putting the heel into the loop of the belt(Step III). This was to make the talus glide in the posterior direction, based on the calf. Here, ankle joint posterior gliding was applied during the restrained dorsiflexion(9). After maintaining this for 10 seconds, the patients took the starting posture again and took a rest for 5 seconds. This was repeated 10 times per set and a total of 6 sets were repeatedly carried out(10, 11).

\section{FES Therapy}

For dorsiflexion necessary for walk of stroke patients, surface electrodes were attached to the fibula nerve and the leg and foot motions were analyzed using WalkAide(Neuromotion, Canada)' sensors and software, while the patients with a foot drop walked. Based on the analysis results, the stimulus timing was determined depending on the gait patterns of individual patients and the tibialis anterior was stimulated for dorsiflexion from the toe-off to the heel strike during walking.

FES was applied for 30 minutes a day and twice a week and for 4 weeks.

\section{A simultaneous application of mobilization and FES}

Mobilization was applied for 15 minutes a day and twice a week and four 4 weeks. After mobilization, for dorsiflexion, the tibialis anterior was stimulated using the surface electrodes attached to the fibula nerve. FES was performed for 15 minutes a day and twice a week and four 4 weeks, as well. Each therapy was performed for 15 minutes to equally apply total 30 minutes of the treatment time to other groups.

Table 1. General characteristics of subjects(M $\pm S D)$

\begin{tabular}{ccccc}
\hline & Mobilization $(\mathrm{n}=8)$ & $\mathrm{FES}(\mathrm{n}=8)$ & Mobilization+FES $(\mathrm{n}=8)$ & $\mathrm{p}$ \\
\hline gender(M/F) & $6 / 2$ & $5 / 3$ & $5 / 3$ & \\
hemiplegia(L/R) & $6 / 2$ & $7 / 1$ & $4 / 4$ & \\
age(y) & $55.25 \pm 11.87$ & $51.25 \pm 13.01$ & $55.00 \pm 14.49$ & .795 \\
height(cm) & $168.57 \pm 7.24$ & $165.83 \pm 8.16$ & $165.27 \pm 7.84$ & .666 \\
weight(kg) & $62.80 \pm 6.55$ & $58.62 \pm 7.37$ & $61.96 \pm 4.82$ & .395 \\
\hline
\end{tabular}




\section{Measurement}

\section{Ankle dorsiflexion PROM}

In a lying position, the patients were asked to bend the knee 20 to 30 degrees. Then, the axis of the Goniometer was placed 1 inch away from the lateral condyle of the fibula and the fixing arms were placed in the lateral centerline in parallel towards the head of the fibula. After positioning the ankle in neutral to keep the two arms at 90 degrees, the patients were asked to move the arms actively to measure the active range of motion. Then, passive dorsiflexion was performed to measure the passive range of motion.

\section{$10 \mathrm{~m}$ walking test}

For the $10 \mathrm{~m}$ walking test, the method with a high intra- and inter-rater reliability $\left(0.89^{\sim} 1.00\right)(12)$ was employed.

\section{Gait analysis}

Using a gail analyzer, G-Walk(BTS Bioengineering, Puerto Rico), gait velocity and stride length were measured.

\section{Analysis}

For data analysis of the study, collected data were statistically analyzed using the SPSS 20.0 for window version. To analyze the general characteristics of the subjects, frequency analysis and descriptive statistics were adopted. To analyze each group before and after the experiments, also, paired t-test was used. One-way ANOVA was conducted to compare the results of the three groups and the significance level was set at .05.

\section{RESULTS}

\section{The effect of the comparative study between the three groups on the dorsiflexion PROM}

This significantly increased in the group of mobilization therapy, mobilization and FES all together( $p<.01)$. There were statistically significant differences among the three groups(p<.01)〈Table 2〉.

\section{The effect of the comparative study between the three groups on the $10 \mathrm{~m}$ walking test}

This significantly decreased in the group of mobilization therapy, mobilization and FES all together(p<.05). There were statistically significant differences among the three groups(p〈.01)〈Table 2$\rangle$.

\section{The effect of the comparative study between the three groups on gait velocity}

This significantly increased in the group of mobilization therapy, FES therapy, mobilization and FES all together( $p<.05)$. There were statistically significant differences among the three groups $(p<.01)<$ Table $2\rangle$.

\section{The effect of the comparative study between the three groups on stride length}

This significantly increased in the group of mobilization therapy, mobilization and FES all together( $\mathrm{p}<.05)$. There were statistically significant differences among the three groups $(p\langle .05)\langle$ Table 2$\rangle$.

Table 2 The effect of the comparative study between the three groups

\begin{tabular}{ccccccc}
\hline \multirow{2}{*}{ Variable } & \multicolumn{2}{c}{ Mobilization } & \multicolumn{2}{c}{ FES } & \multicolumn{2}{c}{ Mobilization+FES } \\
\cline { 2 - 6 } & before & after & before & after & before & after \\
\hline Dorsi Flexion PROM & $1.25 \pm 1.83$ & $4.25 \pm 2.25^{*}$ & $3.37 \pm 2.87$ & $4.25 \pm 1.90 \dagger$ & $1.75 \pm 1.48$ & $4.37 \pm 1.18^{*}$ \\
10M Gait & $15.22 \pm 7.78$ & $11.97 \pm 4.72^{*}$ & $9.59 \pm 4.15$ & $8.20 \pm 2.31$ & $17.33 \pm 6.15$ & $9.53 \pm 2.55^{*} \ddagger$ \\
Gait Velocity & $30.15 \pm 8.91$ & $32.32 \pm 8.09^{*}$ & $47.01 \pm 13.30$ & $51.23 \pm 11.51^{*}$ & $29.58 \pm 21.77$ & $46.45 \pm 30.99^{*} \ddagger \#$ \\
stride length & $1.03 \pm .09$ & $1.12 \pm .07^{*}$ & $1.19 \pm .21$ & $1.17 \pm .11$ & $1.11 \pm .21$ & $1.34 \pm .30^{*} \ddagger$ \\
\hline
\end{tabular}

The values were expressed as the mean \pm standard deviation.

FES, functional electrical stimulation

${ }^{*} \mathrm{p}<.05$ for the change within the group between before and after treatment

$\dagger$ Significant difference between mobilization and FES ( $p<.05)$.

¥ Significant difference between FES and Mobilization+FES (p<.05).

\# Significant difference between Mobilization and Mobilization+FES ( $p<.05)$. 


\section{DISCUSSION and CONCLUSION}

This study was designed to compare and analyze the influence of mobilization and FES on the ankle joint range of motion and the quality of gait when they were simultaneously applied to hemiplegic patients with limited ankle joint motions.

When the dorsiflexion passive range of motion was measured, this significantly increased in the group to perform mobilization therapy, mobilization and FES simultaneously and there were significant differences among the groups. An at al.(7) demonstrated that ankle's passive and active range of motion increased when mobilization with active movement was applied to chronic hemiplegic patients and this corresponds with the finding of this study. It is considered that mobilization improved the mobility and positively influenced ankle joint's range of motion by moving the restrained ankle joint of chronic hemiplegic patients.

The $10 \mathrm{~m}$ walking test and gait velocity changes also showed significant differences. Especially, a significant increase in gait velocity was observed in all the three groups. According to the between-group comparison, the $10 \mathrm{~m}$ walking test and gait velocity both showed significant differences, as well. Kim at al.(5) argued that FES positively influenced the improvement of gait abilities of stroke patients and Son at al.(13) said that mobilization with weight shift training applied to hemiplegic patients improved balance and gait velocity. These findings are similar to this study. Once the ankle movement improved through mobilization, it positively influenced the temporal variables of gait. On the contrary, FES was more effective when applied with mobilization than when it was independently applied. Therefore, it is thought that the simultaneous application of mobilization and FES had a positive impact on the temporal variables of gait.

Stride length significantly increased in the group to perform mobilization therapy, mobilization and FES, at the same time and there were significant differences among the groups. An et al.(7) showed a result similar to this study by demonstrating that mobilization with active movement applied to the ankle of chronic hemiplegic patients revealed significant differences in affected step length, stride length and non-affected stride length. It seems the ankle motion improved by mobilization had a positive effect on walking.

These results show that applying mobilization and FES simultaneously to hemiplegic patients with lim- ited ankle joint motions could bring a more satisfactory result than when applying only one of them.

In conclusion, further research would need to expand the research subjects and research period to find a treatment method, which is more effective for the limited ankle joint of hemiplegic patients and it is considered that the simultaneous application of mobilization and FES could be a new intervention for improving the joint ankle motion and the quality of gait of chronic hemiplegic patients.

\section{REFERENCES}

1. An CM, Won JI. Effects of ankle joint mobilization with movement on lower extremity muscle strength and spatiotemporal gait parameters in chronic hemiplegic patients. Phys Ther Kor 2012; 19(3): 20-30.

2. Cho MS. The effect on ankle joint movement by FES application on tibialis anterior muscle in chronic stroke patients. Journal of the Korean Society of Physical Medicine 2011; 6(3): 277-286.

3. Collins N, Teys P, Vicenzino B. The intial effects of a Mulligan's mobilization with movement technique on dorsiflexion and pain in subacute ankle sprains. Man Ther 2004; 9(2): 77-82.

4. Given JD, Dewald JP, Rymer WZ. Joint dependent passive stiffness in paretic and contralateral limbs of spastic patients with hemiparetic stroke. J Neurol Neurosurg Psychiatry 1995; 59(3): 271279.

5. Harlaar J, Becher JG, Snijders CJ et al. Passive stiffness characteristics of ankle plantar flexors in hemiplegia. Clin Biomech(Bristol, Avon) 2000; 15(4): 261-270.

6. Kaltenborn, Freddy M. Manual mobilization of the joints : the Kaltenborn method of joint examination and treatment. Vol 1, The extremities. 10th ed.

7. Kim BJ, Lee SK, Kim MK. The effects of ankle strength exercise and functional electrical stimulation on the ability of balance control and gait in stroke patients. Journal of Sport and Leisure studies 2007; 31: 921-931.

8. Kim YW, Weon JH, Chung BI. Effects of functional electrical stimulation on gait patterns in stroke patient. KAUTPT 2000; 7(3): 72-80.

9. Son HY, Choi JD. The effect of weight shift training with joint mobilization on balance and gait velocity of hemiplegic patients. Phys Ther . 
Kor 2012; 19(1): 10-18

10. Statistics Korea. Cause-specific death, gender, age-and death, death rate. 2013.

11. Steffen TM, Hacker TA, et al. Age-and genderrelated test performance in community-dwelling elderly people : six-minute walk test, berg balance scale, timed up \& go test and gait speeds. Physical Therapy 2002; 82(2): 128-137.
12. Thilmann AF, Fellows SJ, Ross HF. Biomechanical changes at the ankle joint after stroke. J Neurol Neurosurg Psychiatry 1991; 54(2): 134-139.

13. Vicenzino B, Branjerdporn M, Teys P, et al. Intial changes in posterior talar glide and dorsiflexion of the ankle after mobilization with movement in individuals with recurrent ankle sprain. J Orthop Sport Phys Ther 2006; 36(7): 464-471. 